\title{
SYBR-Green-Based Quantitative Real-Time PCR for Discriminating Between Closely Related Angiostrongylus Cantonensis and A. Malaysisensis
}

Wallop Jakkul

Mahidol University Faculty of Tropical Medicine

Kittipong Chaisiri

Mahidol University Faculty of Tropical Medicine

Naowarat Saralamba

Mahidol University Faculty of Tropical Medicine

Yanin Limpanont

Mahidol University Faculty of Tropical Medicine

Sirilak Dusitsittipon

Mahidol University Faculty of Public Health

Vachirapong Charoennitiwat

Mahidol University Faculty of Tropical Medicine

Urusa Thaenkham ( $\nabla$ urusa.tha@mahidol.ac.th )

Faculty of Tropical Medicine, Mahidol University https://orcid.org/0000-0003-0688-2422

\section{Research}

Keywords: Quantitative real-time PCR, Angiostrongylus cantonensis, A. malaysiensis, Infective stage larva, Achatina fulica, Mitochondrial cytochrome b; Cerebrospinal fluid

Posted Date: June 25th, 2020

DOl: https://doi.org/10.21203/rs.3.rs-37815/v1

License: (c) (i) This work is licensed under a Creative Commons Attribution 4.0 International License.

Read Full License 


\section{Abstract}

Background: Angiostrongylus cantonensis is a well-known pathogen causing human angiostrongyliasis eosinophilic meningitis. Humans, as accidental hosts, are infected by eating undercooked snails containing third-stage larvae. A. malaysiensis is closely related to $A$. cantonensis and has been described as a potential human pathogen. Recently, the two species have been reported to have overlapping distributions in the same endemic area, particularly in the Indochina region. Because of their similar morphological characteristics, misidentification often occurs, particularly of the third-stage larva in the snail intermediate host.

Methods: We designed species-specific primers to mitochondrial cytochrome $b$, which was used as a genetic marker. SYBR-green quantitative real-time PCR (qPCR) was employed to quantitatively detect and identify the third-stage larvae and tissue debris in the cerebrospinal fluid (CSF) of a patient, and to quantify third-stage larvae in the snail Achatina fulica collected from the field.

Results: The newly designed primers were highly specific and sensitive, even when using conventional PCR. SYBR green qPCR quantitatively detected around $10^{-4} \mathrm{ng}$ of genomic DNA from one larva and facilitated the specific detection and identification of parasitic genetic material from the CSF of a patient with angiostrongyliasis. The method also estimated the number of larvae in $A$. fulica and revealed that the primary source of Angiostrongylus infection in the King Rama IX public park study area was $A$. malaysiensis, although, the two Angiostrongylus species each infected $10 \%$ of the snails.

Conclusions: Our SYBR green qPCR method is a useful and inexpensive technique for parasite identification and has sufficient sensitivity and specificity to detect a single larva and simultaneously discriminate between $A$. cantonensis and $A$. malaysiensis. The number of larvae infecting or co-infecting the snail intermediate host can also be estimated. In future research, this qPCR method could be employed in a molecular survey of $A$. cantonensis and $A$. malaysiensis occurrence within intermediate and definitive hosts. The technique should also be applied in a study analyzing CSF specimens from patients with eosinophilic meningitis to assess the usefulness of the method for clinical diagnosis.

\section{Background}

The genus Angiostrongylus Kamensky, 1905, contains the zoonotic nematode parasites of vertebrates commonly known as "lungworms" [1]. Several species in this genus have been reported to be human pathogens, including $A$. cantonensis and A. costaricensis, while A. mackerrasae and A. malaysiensis are considered potential human pathogenic parasites [2-5]. Among these species, $A$. cantonensis is a wellknown pathogen that causes angiostrongyliasis eosinophilic meningitis in humans [6], over 2,800 cases of which have been reported worldwide, with a particularly high incidence in Thailand [7]. Humans are accidental hosts and are infected by eating undercooked snails, slugs, paratenic hosts, and contaminated fresh vegetables containing the infective third-stage larvae [8]. The clinical symptoms can range from headaches to coma depending on the number of parasitic larvae [2, 8-10]. Many species of terrestrial 
and freshwater snails, for example, Achatina fulica, Cryptozona spp., Pomacea spp., and Pila spp., are reported to be critical vectors of $A$. cantonensis [11-13]. Global parasite transmission frequency has been promoted by the widespread distribution of some invasive vector species, for example, $A$. fulica and Pomacea spp. [13-15]. The ability of the parasite to spread throughout the world may also be influenced by its natural definitive host, a rodent that inhabits areas of human urbanization $[1,6,16]$.

Among species of the genus Angiostrongylus, a cryptic species of $A$. cantonensis was recently correctly described as $A$. malaysiensis $[17,18]$. Previously, A. malaysiensis was known as the Malaysian strain of $A$. cantonensis $[1,5]$; however, its pathology differed from the typical pathology of $A$. cantonensis through its ability to migrate to the central nervous system of the monkey host but not cause death $[1,19]$. The strain was known to immunize monkeys against the lethal effects of a high number of $A$. cantonensis larvae. Subsequently, the Malaysian strain was species delimited as $A$. malaysiensis $[5,20]$.

The adult stages of $A$. cantonensis and $A$. malaysiensis can be morphologically discriminated by the structure of the bursal rays of males and the minute protrusions at the posterior of females [21]. The third-stage larvae of $A$. cantonensis are described as using the mellifluous "Q"-movement, which clearly distinguishes them from free-living nematodes [22]. However, differences between the morphological characteristics of the larval stage $A$. cantonensis and $A$. malaysiensis have not yet been described.

Previously, the morphological variations between these two species were never considered, and their similarities were the cause of the misidentification. A. cantonensis was known as the predominant species in the genus of Angiostrongy/us, with distribution throughout many countries in Indochina [23]. In 2016, Rodpai et al. revealed that $A$. cantonensis is widely distributed in Thailand, Lao PDR, Cambodia, and Myanmar, and its distribution broadly overlapped that of $A$. malaysiensis [23]. Dusitsittipon et al. discussed the incongruence between the morphological and molecular identifications and found that most of the misidentified specimens were from Thailand $[17,24]$.

An under-estimation of the prevalence of $A$. malaysiensis has occurred because of the difficulty in the morphological identification of adult and third-stage larva. Several polymerase chain reaction (PCR)based identification techniques were developed, but all were aimed at increasing the sensitivity and specificity of $A$. cantonensis detection in blood, the peripheral tissues of wild Hawaiian rats, and cerebrospinal fluid (CSF) of patients [25-27]. No powerful techniques with high sensitivity and specificity have been developed for detecting and discriminating between $A$. cantonensis and A. malaysiensis.

A. cantonensis and $A$. malaysiensis can be distinguished using the mitochondrial cytochrome $b(C y t b)$ gene as a genetic marker because of the robust phylogenetic relationship between the closely related species [17, 24]. This gene sequence shows sufficiently high genetic variation between species, and species-specific primers can be used to detect and discriminate between the two species with high sensitivity and specificity. The SYBR-green quantitative real-time PCR method (qPCR) was considered suitable for quantitative detection and species discrimination because it is cheaper than TaqMan qPCR, which requires a fluorescent probe. SYBR green qPCR has recently been used in many studies to detect, discriminate, and quantify species as, for example, in the discrimination and quantification of Leishmania 
in human samples and the discrimination of species and subspecies of Salmonella. The technique was also employed for the detection of Opisthorchis viverrini and Haplorchis taichui from human stool samples [28-30].

In this study, therefore, we designed species-specific primers to Cytb partial gene sequences, which have sufficiently high genetic variation between species to prevent cross-amplification [17, 24]. We aimed to develop a highly sensitive and specific method capable of quantitively detecting the third-stage larvae of A. cantonensis and A. malaysiensis in their intermediate host, Achatina fulica, and to determine the efficacy of the method for the detection of Angiostrongylus genomic material within the CSF of infected patients.

\section{Methods}

\section{Specimens used}

Reference specimens for evaluating sensitivity and specificity

Third-stage larvae of $A$. cantonensis, adult worms of $A$. cantonensis and $A$. malaysiensis, CSF of patients with neurocysticercosis, gnathostomiasis, and angiostrongyliasis, and negative CSF of angiostrongyliasis were obtained from archived research specimen stock kept in the Department of Helminthology, Mahidol University. The selected CSF was from patients with diseases related to eosinophilic meningitis. The larvae and adult worms of Angiostrongylus were preserved in $70 \%$ ethanol at $-20^{\circ} \mathrm{C}$, while the CSF specimens were kept at $-80^{\circ} \mathrm{C}$. The specimens were used to test the sensitivity and specificity of the species-specific primers designed for the SYBR green qPCR.

\section{Experimental specimens to determining the validity of SYBR green qPCR}

Third-stage larvae of Angiostrongylus were collected from 48 A. fulica collected at King Rama IX Public Park in Bangkok, Thailand (geographical coordinates 13.68N, 100.65E). The snails were transported to the laboratory of the Department of Helminthology within 1 hour. The snails were then euthanized at $0^{\circ} \mathrm{C}$ for $10 \mathrm{~min}$. Then, the shell of each snail was removed with a meat grinder. The foot and mantel parts of the snail were dissected and individually incubated with a digestion solution ( $1 \% \mathrm{HCl}$ and $1 \% \mathrm{Pepsin})$ at $37^{\circ} \mathrm{C}$ for 1 hour. The digestion method used for the snails followed the protocol of Vitta et al.[31]. The larvae collected were identified as Angiostrongylus according to the morphological criteria described by Ash [32]. The larvae from each positive snail were approximately counted and preserved in $70 \%$ ethanol at $-20^{\circ} \mathrm{C}$ until used for DNA extraction. The wild snail collection was performed with permission from the Animal Care and Use Committee, Faculty of Tropical Medicine, Mahidol University, Bangkok (No. FTMACUC 024/2018).

\section{Preparation of standard genomic DNA}


The individual adult $A$. cantonensis and $A$. malaysiensis of the described reference specimens were transferred into a 1.7-ml centrifuge tube and washed thoroughly with sterile distilled water. Total genomic DNA (gDNA) was extracted from each specimen using the Genomic DNA mini kit (Geneaid Biotech Ltd, Taipei, Taiwan) following the manufacturer's instructions. The gDNA was eluted from the column with 30 $\mu \mathrm{l}$ of PCR-grade sterile water and the concentration measured using a NanoDrop ${ }^{\mathrm{TM}} 1000$ spectrophotometer (Thermo Fisher Scientific, MA, USA). The gDNA was stored at $4{ }^{\circ} \mathrm{C}$ until use.

\section{Design of species-specific primers}

Species-specific primers were designed manually based on the $C y t b$ gene sequences of $A$. cantonensis (GenBank accession numbers KC995211, KC995223, KC995262) and A. malaysiensis (GenBank accession numbers KX147380, KX147406, KX147442). The properties of the oligonucleotide primers, including GC content, amplicon size, melting temperature, and hairpin formation, were predicted by OligoCalc version 3.27 and Primer3 [33, 34]. Information on the designed primers is shown in Table S1.

\section{Optimization of PCR conditions using conventional PCR}

Optimization of the PCR conditions was performed prior to the main quantitative reaction using conventional PCR, and the sensitivity and specificity of the newly designed primers were evaluated. Afterward, the optimized conditions for the species-specific primers of $A$. cantonensis and $A$. malaysiensis were applied in the SYB-green qPCR.

\section{Sensitivity test}

The gDNA of adult $A$. cantonensis and $A$. malaysiensis reference specimens were prepared by 10 -fold serial dilution (between $10^{-5}$ and $1 \mathrm{ng} / \mu \mathrm{l}$ ) for use as DNA templates. The $20 \mu \mathrm{l} \mathrm{PCR}$ mixture contained 1 $\times$ One PCR ${ }^{\text {TM }}$ Plus mixture (GeneDireX Inc., Taoyuan, Taiwan) $10 \mu \mathrm{M}$ of species-specific primers and $1 \mu \mathrm{l}$ of gDNA template. The PCR was performed with an initial denaturation at $95^{\circ} \mathrm{C}$ for 5 min, followed by 34 cycles of denaturation at $95^{\circ} \mathrm{C}$ for $30 \mathrm{~s}$, gradient primer annealing between $55^{\circ} \mathrm{C}$ and $60^{\circ} \mathrm{C}$ for $30 \mathrm{~s}$, extension at $72^{\circ} \mathrm{C}$ for $30 \mathrm{~s}$, and final elongation at $72^{\circ} \mathrm{C}$ for $5 \mathrm{~min}$. The reaction mixtures were then held at $12^{\circ} \mathrm{C}$ until the PCR products were collected. The PCR was conducted on a T100 ${ }^{\mathrm{Tm}}$ thermocycler (Bio-Rad Laboratories, CA, USA). The PCR products were run on $2 \%$ agarose gel at $50 \mathrm{~V}$ for 1 hour to determine the size of the amplicons.

\section{Specificity test}

Ten-fold serial dilutions of the gDNA from the reference specimens $A$. cantonensis and A. malaysiensis were used to determine the specificity of the primers. The DNA templates were used in an experimental mixture of species using different ratios of gDNA of $A$. cantonensis and A. malaysiensis, as described in Table S2. This was used to evaluate the robustness of further SYBR-green qPCRs. DNA amplification for the specificity test was performed following the PCR conditions described for the sensitivity test. The PCR products were run on $2 \%$ agarose gel at $50 \mathrm{~V}$ for 1 hour and stained with SYBR ${ }^{\mathrm{TM}}$ Safe (Life Technologies, CA, USA) to determine the species-specific band sizes of $A$. cantonensis and A. malaysiensis. The PCR 
amplicons were then sequenced by the Sanger method with the PCR primers (Macrogen Inc., Seoul, Korea).

\section{SYBR-green Quantitative Real-time PCR}

The SYBR-green qPCR reactions were performed separately for each species using the species-specific primers. The qPCR was performed following the protocol of the Luna ${ }^{\circledR}$ Universal qPCR master mix (New England Biolabs, MA, USA). The reactions contained $10 \mu$ of the master mix, $10 \mu \mathrm{M}$ of each pair of species-specific primers, and $1 \mu \mathrm{l}$ of gDNA, and RNAase-free water was added to a final volume of $20 \mu \mathrm{l}$. The reaction was performed on a CFX96 TouchTM Real-Time PCR machine (Bio-Rad Laboratories, CA, USA) with the standard PCR conditions. The real-time PCR thermal cycle included an initial denaturation at $95^{\circ} \mathrm{C}$ for $60 \mathrm{~s}$, followed by 45 cycles of denaturation at $95^{\circ} \mathrm{C}$ for $15 \mathrm{~s}$, and an extension at $60^{\circ} \mathrm{C}$ for $30 \mathrm{~s}$. A final melting analysis program was applied at $60^{\circ} \mathrm{C}$ to $95^{\circ} \mathrm{C}$ and the duration of each cycle was $5 \mathrm{~s}$ with a $0.5^{\circ} \mathrm{C}$ increment per cycle. The SYBR-green qPCR described above was used for standard curve preparation, specificity and sensitivity assays, reproducibility assay, and implementation of the developed method to detect the third-stage larvae of Angiostrongylus in A. fulica.

\section{Standard curve construction for SYBR-green qPCR}

Ten-fold serial dilutions of $A$. cantonensis and $A$. malaysiensis adult worm gDNA were used to construct the SYBR-green qPCR standard curve; gDNA concentrations of $10^{-4}$ to $1 \mathrm{ng} / \mu \mathrm{l}$ were used as DNA templates for qPCR, and each DNA concentration was amplified three times. The precision of the standard curve and robustness of the GPCR were verified by considering the slope values, correlation coefficient, and qPCR efficiency of both $A$. cantonensis and A. malaysiensis.

\section{Specificity assay}

The species-specific primers developed for $A$. cantonensis and $A$. malaysiensis were evaluated for specificity to the various gDNA templates described in Table 2 using the SYBR-green qPCR profile described above. The qPCRs were conducted three times for each gDNA template. The specificity of the qPCR was also tested with the CSF of patients with gnathostomiasis, cysticercosis, and angiostrongyliasis. The PCR products were then run on $2 \%$ agarose gel at $50 \mathrm{~V}$ for 1 hour and stained with SYBR ${ }^{\text {TM }}$ Safe (Life Technologies, CA, USA) to determine the species-specific band sizes with the positive controls for each Angiostrongy/us species. The purified DNA samples were sequenced by Macrogen (Seoul, South Korea), an external biotechnology company, using the Sanger sequencing method with the primers used for the PCR amplification. The obtained nucleotide sequences (query sequences) were confirmed as the specific species target sequences by comparing with annotated sequences in the NCBI databases using the standard nucleotide BLAST [35]. 
Table 2

Intra- and inter-reproducibility assays of qPCR for the species-specific primers of A. malaysiensis.

\begin{tabular}{|cllll|}
\hline Category & Genomic DNA standard $(\mathrm{ng} / \mu \mathrm{l})$ & Mean $(\mathrm{Ct})$ & $\mathrm{SD}$ & $\mathrm{CV}(\%)$ \\
\hline Intra-assay & $1 \times 10^{1}$ & 15.65 & 0.077 & 0.492 \\
& $1 \times 10^{-1}$ & 19.32 & 0.019 & 0.098 \\
& $1 \times 10^{-2}$ & 22.33 & 0.052 & 0.233 \\
\hline $1 \times 10^{-3}$ & 26.12 & 0.048 & 0.184 \\
\hline $1 \times 10^{-4}$ & 29.31 & 0.017 & 0.058 \\
\hline $1 \times 10^{1}$ & 15.40 & 0.354 & 2.299 \\
\hline $1 \times 10^{-1}$ & 18.99 & 0.467 & 2.459 \\
\hline $1 \times 10^{-2}$ & 22.24 & 0.134 & 0.603 \\
\hline $1 \times 10^{-3}$ & 26.08 & 0.057 & 0.219 \\
\hline $1 \times 10^{-4}$ & 29.02 & 0.410 & 1.413 \\
\hline
\end{tabular}

\section{Sensitivity assay}

To test the sensitivity of the SYBR-green qPCR method, we used gDNA from groups of $1,5,10,50,100$, and 200 third-stage $A$. cantonensis larvae. Before gDNA isolation, the cuticles of the larvae were broken by bead beating with $20 \mathrm{mg}$ of $0.1 \mathrm{~mm}$ silica beads in $200 \mu$ lysis buffer using Tissue Lyser LT at $50 \mathrm{~Hz}$ for $30 \mathrm{~s}$ (Qiagen, Hilden, Germany). Each larval sample was beaten three times and then left on ice to prevent DNA degradation. The gDNA from each group of larvae was then extracted using the Tissue Genomic DNA Mini kit (Geneaid Biotech Ltd, Taipei, Taiwan). Subsequently, each sample was amplified three times with the SYBR-green qPCR profile described above. The threshold cycle $\left(\mathrm{C}_{t}\right)$ or the quantitation cycle $(\mathrm{Cq})$ values obtained for each group were estimated with the standard curve for the qPCR to determine the amount of larval gDNA.

\section{Reproducibility assay}

The 10-fold serial dilution of the standard DNA, which was used to construct the standard curve, was used in the reproducibility assay. The intra-reproducibility assay was estimated by amplifying three replicates of each DNA concentration using the qPCR profile described above. Variations in the intrareproducibility assays were assessed. The experiments were repeated on 2 different days to determine the inter-reproducibility. The mean, SD, and coefficient of variation (CV) were calculated separately using the $\mathrm{Ct}$ values to evaluate the reproducibility of the developed SYBR-green qPCR. The percentage of the CV of 
inter- and intra-reproducibility assays were calculated to determine the consistency of the assay manipulation.

Detection of third-stage larvae of A. cantonensis and A. malaysiensis from naturally infected A. fulica

The developed SYBR-green qPCR was implemented to detect and identify the third-stage larvae of Angiostrongylus collected from A. fulica. Before gDNA extraction, the larval cuticles were disrupted as described above. The larvae were then removed separately from each snail, and the DNA was extracted using the tissue genomic DNA mini kit (Geneaid, Taipei, Taiwan) according to the manufacturer's instructions. The extracted gDNA was then used as the template in the developed qPCR method. The standard curves were constructed to estimate the number of larvae and the ratio of $A$. cantonensis to $A$. malaysiensis in each $A$. fulica.

\section{Results}

\section{Optimization of the newly designed species-specific primers using conventional PCR}

\section{Sensitivity and specificity tests with conventional PCR}

The PCR products from the 10-fold diluted gDNA of $A$. cantonensis and A. malaysiensis are shown in Figure $\mathrm{S} 1$. The different sizes of the species-specific bands were used to discriminate between $A$. cantonensis (117 bp) and A. malaysiensis (141 bp). The amplifications were successful for DNA concentrations of $10^{-4}$ to $1 \mathrm{ng} / \mu \mathrm{l}$.

The mixed $A$. cantonensis and $A$. malaysiensis gDNA was used to evaluate the specificity of the newly designed species-specific primers. Different ratios of gDNA from the two species, including $10^{-2}: 10^{-1} \mathrm{ng}$, and vice versa, and 1:1 ng were amplified with conventional PCR using the above conditions. The sizes of the species-specific PCR amplicons were used to determine the specificity of the designed primers (Fig. S2). The DNA sequences of those PCR amplicons also confirmed the PCR specificity.

\section{Standard curve for SYBR-green quantitative real-time PCR}

The effectiveness of the SYBR-green qPCR was determined using the species-specific primers for $A$. cantonensis and $A$. malaysiensis Cytb. The standard curves for $A$. cantonensis (Slope $=-3.379, \mathrm{R}^{2}=$ 0.999 ) and $A$. malaysiensis (Slope $=-3.412, \mathrm{R}^{2}=0.999$ ) are shown in Fig. 1 . The efficiency values of the qPCR for both species-specific primers of $A$. cantonensis and $A$. malaysiensis were $94.8 \%$ and $96.4 \%$, respectively.

\section{Sensitivity and specificity of SYBR green quantitative real- time PCR}


The sensitivities of the species-specific primers used in SYBR-green qPCR were tested using the gDNA extracted from various $A$. cantonensis larvae group sizes from 1 to 200 . The $C q$ values for the amplified larval gDNA were compared with those of the standard curve. Approximately $1 \mathrm{ng}$ of gDNA was extracted from 100 larvae, while $10^{-4}$ ng of gDNA was extracted from 1 larva (Fig. 2A). The qPCR melting curve and the gel electrophoresis demonstrated the specificity of the primers to the $A$. cantonensis gDNA (Fig. 2B).

The specificity of the SYBR-green qPCR was determined using the gDNA template described in Table S2. Figure $\mathrm{S} 3$ shows the specificity of the $A$. cantonensis and $A$. malaysiensis-specific primers. The speciesspecific primers did not amplify the gDNA across different species. The high specificities of the AC4_cytb_F and AC5_cytb_R primers for $A$. cantonensis were also demonstrated for the artificially mixed A. cantonensis and $A$. malaysiensis gDNA, in both the $1: 1$ ratio and when there was less $A$. cantonensis than $A$. malaysiensis gDNA. These primers did not amplify $C y t b$ from gnathostomiasis and neurocysticercosis samples or the heterogeneous sample containing $1 \mathrm{ng}$ of $A$. malaysiensis gDNA. The specificity of the AC4_cytb_F and AC5_cytb_R primers was confirmed by the size of the qPCR amplicon (Fig. 3A). A specificity assay was then performed with AM3_cytb_F and AM4_cytb_R primers for $A$. malaysiensis. They also showed a specificity similar to the primers for $A$. cantonensis. The primers designed for $A$. malaysiensis did not amplify any Cytb from the CSF of patients, including those with angiostrongyliasis (Fig. 3B).

\section{Intra- and inter-reproducibility assays of SRBR-green qPCR}

Intra- and inter-reproducibility assays were performed similarly to the method used to construct the $A$. cantonensis and A. malaysiensis gDNA standard curves. Serial concentrations of gDNA from $10^{-4}$ to $1 \mathrm{ng}$ were amplified using SYBR-green qPCR. Three replicates of each DNA concentration were conducted for the intra-reproducibility assay, and the qPCRs were repeated over 3 days. The results showed there was consistent amplification between the replicated series of gDNA concentrations in the same assay (inter-reproducibility). The precision of the assay manipulation was considered to be the reproducibility of the qPCR reactions for $A$. cantonensis and $A$. malaysiensis, which is shown in Tables 1 and 2. 
Table 1

Intra- and inter-reproducibility assays of qPCR for the species-specific primers of

A. cantonensis.

\begin{tabular}{|lllll|}
\hline Category & Genomic DNA standard $(\mathrm{ng} / \mu \mathrm{l})$ & Mean $(\mathrm{Ct})$ & $\mathrm{SD}$ & $\mathrm{CV}(\%)$ \\
\hline Intra-assay & $1 \times 10^{1}$ & 15.63 & 0.051 & 0.326 \\
\hline $1 \times 10^{-1}$ & 19.33 & 0.103 & 0.533 \\
& $1 \times 10^{-2}$ & 22.38 & 0.012 & 0.054 \\
\hline $1 \times 10^{-3}$ & 26.21 & 0.065 & 0.248 \\
\hline $1 \times 10^{-4}$ & 29.46 & 0.168 & 0.570 \\
\hline $1 \times 10^{1}$ & 15.53 & 0.148 & 0.953 \\
\hline $1 \times 10^{-1}$ & 19.26 & 0.099 & 0.514 \\
\hline $1 \times 10^{-2}$ & 22.35 & 0.049 & 0.219 \\
\hline $1 \times 10^{-3}$ & 26.30 & 0.120 & 0.456 \\
\hline $1 \times 10^{-4}$ & 29.49 & 0.035 & 0.119 \\
\hline
\end{tabular}

Detection of the third-stage Angiostrongylus larvae from naturally infected A. fulica

The SYBR-green qPCR results showed that the species-specific primers discriminated between $A$. cantonensis and A. malaysiensis third-stage larvae. All of the third-stage Angiostrongylus larvae collected from each snail were approximately counted, and DNA was extracted without excluding dead and weakened larvae. Larvae numbers in each snail were estimated as the degree of intensity, and the results are shown in Table 3. The qPCR results suggested there was no cross-amplification. The Angiostrongylus larvae detected from four infected snails were mainly $A$. malaysiensis, while two snails had coinfections of $A$. malaysiensis and A. cantonensis. There were fewer Angiostrongylus larvae counted from each snail than the numbers estimated by qPCR. 
Table 3

The SYBR green qPCR results of the Angiostrongylus larvae infected in four from fourty-eight $A$. fulica snails collected from King Rama IX Park, Bangkok, Thailand.

\begin{tabular}{|lllll}
\hline $\begin{array}{l}\text { A. fulica } \\
\text { snail }\end{array}$ & $\begin{array}{l}\text { Angiostrongylus larva } \\
\text { intensity }\end{array}$ & \multicolumn{2}{l}{$\begin{array}{l}\text { Ct values of SYBR green } \\
\text { qPCR }\end{array}$} & $\begin{array}{l}\text { Estimated larva } \\
\text { numbers }\end{array}$ \\
\cline { 3 - 5 } & & $\begin{array}{l}\text { A. } \\
\text { cantonensis } \\
\text { (Ac) }\end{array}$ & $\begin{array}{l}\text { A. } \\
\text { malaysiensis } \\
\text { (Am) }\end{array}$ & \\
\hline AF_P_01 & +++ & 34.32 & 21.30 & Ac $\geq 1$ \\
& & & & Am $\geq 50$ \\
\hline AF_P_02 & + & 35.42 & 22.97 & Ac $\geq 1$ \\
\hline AF_P_03 & +++ & & & $\mathrm{Am} \geq 50$ \\
\hline AF_P_04 & + & & 29.13 & $\mathrm{Am} \geq 1$ \\
\hline
\end{tabular}

\section{Discussion}

In this study, we developed a simple, economical, highly sensitive, and specific assay based on SYBRgreen qPCR for the detection, discrimination, and quantification of third-stage larvae of $A$. cantonensis and its closely related species $A$. malaysiensis from the intermediate host $A$. fulica. Recently, in some areas, and particularly areas in Indochina, there have been reports of overlap in the distributions of $A$. cantonensis and A. malaysiensis within the terrestrial snail host [23]. Although the gold standard for Angiostrongylus identification is the use of morphological characteristics, the characteristics of the thirdstage larvae of these two species are very similar $[5,21,32]$ and misidentification often occurs.

We designed species-specific primers to the mitochondrial $C y t b$ gene of $A$. cantonensis and $A$. malaysiensis based on the genetic variation in the partial sequences. The two species can be differentiated by comparing the size of conventional PCR products for mitochondrial $C y t b$ on gel electrophoresis (see Fig. S1). The high sensitivity of conventional PCR allows the amplification of $10^{-4}$ ng of gDNA. The newly designed primers showed high specificity without cross-species amplification when a heterogeneous gDNA sample containing a small amount of specific DNA template was used (Fig. S2). However, conventional PCR cannot quantify the number of Angiostrongylus larvae. Therefore, a qPCR method was developed that could detect a small amount of DNA $\left(10^{-4} \mathrm{ng}\right.$ or less) from both Angiostrongylus species. We also established the standard curve and demonstrated the high efficiency of the qPCR reactions (see Fig. $1 \mathrm{~A}$ and $\mathrm{B}$ ). The \% CV of reproducibility confirmed that the standard curve construction had low system variation in the manipulation assay. The number of larvae was then estimated using a standard curve constructed from 10-fold serial DNA dilutions. Based on the qPCR results, we postulated that DNA damage and loss during the larvae preservation and DNA extraction 
process might have resulted in the fluctuation of the Cq value, particularly for a small amount of DNA. In contrast, there was no effect seen when more than 10 larvae were used (Fig. 2A).

We also confirmed the high specificity and absence of cross-amplification of the method using heterogeneous gDNA in the SYBR-green qPCR reactions (Fig. 3). The developed method provides an alternative way of detecting the low amount of Angiostrongy/us genetic material contained in CSF specimens. We also showed the potential of using SYBR-green qPCR for diagnosis using the CSF of the patients with an $A$. cantonensis infection, and there was no cross-reaction with other diseases that cause eosinophilic meningitis, such as cysticercosis and gnathostomiasis.

After proving the efficacy and consistency of the developed qPCR assay, we implemented it in the molecular discrimination of $A$. cantonensis and A. malaysiensis third-stage larvae from $A$. fulica collected in a suburban public park in Bangkok, Thailand. The number of larvae can be ascertained using the developed qPCR method and comparing the results to the standard curve (see Fig. 2A). We obtained relatively fewer numbers from the larvae count compared with the qPCR estimate because the larvae collected from the snails included those that were dead and dying. Therefore, when applying this method to a molecular survey of Angiostrongylus larvae, the report should indicate that the numbers obtained are approximate or only indicate the ratio between species. When small numbers of larvae are being studied and species discrimination without quantification is adequate, we suggest that conventional PCR is used with the species-specific primers.

Although the developed method required the design of two species-specific primers, which were tested using two separate DNA templates, the two sets of primers can be used together with the same qPCR conditions and are sensitive enough to detect a single target species. This method does not require the design of a separate probe, such as with the TaqMan qPCR technique, which can be complicated and expensive. Moreover, SYBR green qPCR appears to be more sensitive than the TaqMan probe-based qPCR $[29,36]$. The specificity of SYBR-green qPCR may also provide information regarding the amount of DNA amplification by using the melting curve analysis [37].

Previously, the TaqMan probe real-time PCR was developed using ribosomal internal transcribed spacer 1 as the genetic marker [38] for detecting third-stage $A$. cantonensis larvae in mollusks, and it was more sensitive than 18S rDNA-based conventional PCR [38, 39]. However, the TaqMan qPCR was not designed to estimate larval numbers or the level of infection in the snails [38]. The technique of detecting the DNA of $A$. cantonensis was applied to the diagnoses of patients with eosinophilic meningitis [26]. Although the newly developed SYBR-green qPCR method, using the mitochondrial Cytb gene as a marker, has not yet been proven to be suitable for molecular diagnosis, it may provide an alternative method in the future. The new technique has the potential to confirm the occurrence of $A$. malaysiensis infection in patients living in areas where the distributions of $A$. cantonensis and $A$. malaysiensis overlap.

Although the sensitivity assay for the developed qPCR was limited because of the lack of a verified source of $A$. malaysiensis larvae, such as a reference strain, an assessment of the amount of DNA from various numbers of $A$. cantonensis was used instead. The high precision of the technique was clear, as 
the mean $\mathrm{Ct}$ values for $A$. cantonensis and $A$. malaysiensis were similar between manipulation assays, even for low amounts of gDNA (Tables 3 and 4). This evidence can be used to infer that the approximate amount of gDNA from the various $A$. cantonensis larva numbers can be used to represent $A$.

malaysiensis. The approximate number of $A$. malaysiensis larvae may be determined from the estimated number in the sensitivity test of $A$. cantonensis larvae (Fig. 2A). The developed method has not yet been employed to detect larvae in $A$. fulica tissue quantitively. We suggest that larvae should be isolated from the snails before gDNA extraction to determine the accuracy of the larvae number estimated by the qPCR method.

\section{Conclusions}

The developed SYBR-green GPCR method is a useful and inexpensive alternative technique for molecular parasite detection. It has sufficiently high sensitivity and specificity to simultaneously detect a single larva of Angiostrongylus and discriminate between larvae of $A$. cantonensis and $A$. malaysiensis. The developed qPCR method can also be used to estimate the number or ratio of larvae infecting or coinfecting the snail intermediate host. In further studies, the developed qPCR method may be used to perform a molecular survey of the incidence of $A$. cantonensis and $A$. malaysiensis in the natural intermediate host and the definitive rodent host, particularly in Indochina, which has been found to be an area with overlapping distributions of $A$. cantonensis and $A$. malaysiensis. However, the suitability of the method for diagnosis in the clinical setting needs to be evaluated by testing mixtures of unknown parasitic and free-living nematode larvae. In the future, the usefulness of the developed SYBR-green qPCR technique for clinical diagnosis using CSF specimens from patients with eosinophilic meningitis should also be assessed.

\section{Declarations}

\section{Ethics approval and consent to participate}

Ethical clearance was provided by the Animal Care and Use Committee, Faculty of Tropical Medicine, Mahidol University, Bangkok (No. FTM-ACUC 024/2018)

\section{Consent for publication}

Not applicable

\section{Availability of data and material}

All data generated during this study are included in the published article

\section{Competing interests}

Authors declare that they have no competing interests 


\section{Funding}

This research did not receive any specific grant from funding agencies in the public, commercial, and notfor-profit sectors.

\section{Authors' contributions}

UT, KC, NS, YL conceived the study. WJ performed molecular works. WJ, KC, SD, VC, UT collected the samples. All authors reviewed the manuscript draft and read and approved the final manuscript.

\section{References}

1. Spratt DM. Species of Angiostrongylus (Nematoda: Metastrongyloidea) in wildlife: A review. Int J Parasitol Parasites Wildl. 2015;4:178-89.

2. Ansdell V, Wattanagoon Y. Angiostrongylus cantonensis in travelers: clinical manifestations, diagnosis, and treatment. Curr Opin Infect Dis. 2018;31:399-408.

3. Aghazadeh M, Harvie MC, Owen HC, Verissimo C, Aland KV, Reid SA, et al. Comparative pathogenesis of eosinophilic meningitis caused by Angiostrongylus mackerrasae and Angiostrongylus cantonensis in murine and guinea pig models of human infection. Parasitol. 2016;143:1243-51.

4. Sawabe K, Makiya K. Genetic variability in isozymes of Angiostrongy/us malaysiensis. Southeast Asian J Trop Med. 1994;25:728-36.

5. Bhaibulaya M, Cross J. Angiostrongylus malaysiensis (Nematoda: Meta-strongylidae), a new species of rat lung-worm from Malaysia. Southeast Asian J Trop Med. 1971;2:527-33.

6. Barratt J, Chan D, Sandaradura I, Malik R, Spielman D, Lee R, et al. Angiostrongylus cantonensis: a review of its distribution, molecular biology and clinical significance as a human pathogen. Parasitol. 2016;143:1087-118.

7. Eamsobhana P. Angiostrongyliasis in Thailand: epidemiology and laboratory investigations. Hawaii J Med Public Health. 2013;72(Suppl 2):28.

8. Wang Q-P, Wu Z-D, Wei J, Owen R, Lun Z-R. Human Angiostrongylus cantonensis: an update. Eur J Clin Microbiol Infect Dis. 2012;31:389-95.

9. Pozio E. Foodborne nematodes. In: Gajadhar AA, editor. Foodborne Parasites in the Food Supply Web. Cambridge: Woodhead Publishing; 2015. pp. 165-99.

10. Johnston DI, Dixon MC, Elm JL Jr, Calimlim PS, Sciulli RH, Park SY. Review of cases of angiostrongyliasis in Hawaii, 2007-2017. Am J Trop Med Hyg. 2019;101:608-16.

11. Vitta A, Polsut W, Fukruksa C, Yimthin T, Thanwisai A, Dekumyoy P. Levels of infection with the lungworm Angiostrongylus cantonensis in terrestrial snails from Thailand, with Cryptozona siamensis as a new intermediate host. J Helminthol. 2016;90:737-41.

12. Hu Q-A, Zhang Y, Guo Y-H, Lv S, Xia S, Liu H-X, et al. Small-scale spatial analysis of intermediate and definitive hosts of Angiostrongylus cantonensis. Infect Dis Poverty. 2018;7:100. 
13. Thiengo S, Maldonado A, Mota EM, Torres EJL, Caldeira R, Carvalho OdS, et al. The giant African snail Achatina fulica as natural intermediate host of Angiostrongylus cantonensis in Pernambuco, northeast Brazil. Acta Trop. 2010;115:194-9.

14. Fontanilla I, Wade C. Research Note: First report of Angiostrongylus cantonensis in the giant African land snail Achatina fulica in French Polynesia detected using the SSU rRNA gene. Trop Biomed. 2012;29:642-5.

15. Yang T-B, Wu Z-D, Lun Z-R. The apple snail Pomacea canaliculata, a novel vector of the rat lungworm, Angiostrongylus cantonensis: its introduction, spread, and control in China. Hawaii J Med Public Health. 2013;72(Suppl 2):23.

16. Alicata JE. Biology and distribution of the rat lungworm, Angiostrongylus cantonensis, and its relationship. In: to eosinophilic meningoencephalitis and other neurological disorders of man and animals. Adv Parasitol. London: Academic Press; 1965. pp. 223-48.

17. Dusitsittipon S, Criscione CD, Morand S, Komalamisra C, Thaenkham U. Cryptic lineage diversity in the zoonotic pathogen Angiostrongylus cantonensis. Mol Phylogenet Evol. 2017;107:404-14.

18. Eamsobhana P, Lim P, Yong H. Phylogenetics and systematics of Angiostrongy/us lungworms and related taxa (Nematoda: Metastrongyloidea) inferred from the nuclear small subunit (SSU) ribosomal DNA sequences. J Helminthol. 2015;89:317-25.

19. Cross JH, Chen E. Angiostrongyliasis. Food-borne parasitic zoonoses. New York: Springer; 2007. pp. 263-90.

20. Cross J, Bhaibulaya M. Validity of Angiostrongylus malaysiensis Bhaibulaya and Cross, 1971. Southeast Asian J Trop Med. 1974;5:374.

21. Bhaibulaya M. Morphology and taxonomy of major Angiostrongylus species of Eastern Asia and Australia. Studies on Angiostrongylus in Eastern Asia and Australia Taipei, Taiwan. Maryland: US Naval Medical Research Unit 2; 1979. p. 4-13.

22. Lv S, Zhang Y, Liu H-X, Hu L, Yang K, Steinmann P, et al. Invasive snails and an emerging infectious disease: results from the first national survey on Angiostrongylus cantonensis in China. PLOS Neglect Trop Dis. 2009;3:e368.

23. Rodpai R, Intapan PM, Thanchomnang T, Sanpool O, Sadaow L, Laymanivong S, et al. Angiostrongylus cantonensis and A. malaysiensis broadly overlap in Thailand, Lao PDR, Cambodia and Myanmar: a molecular survey of larvae in land snails. PLoS One. 2016;11:e0161128.

24. Dusitsittipon S, Criscione CD, Morand S, Komalamisra C, Thaenkham U. Hurdles in the evolutionary epidemiology of Angiostrongylus cantonensis: pseudogenes, incongruence between taxonomy and DNA sequence variants, and cryptic lineages. Evol Appl. 2018;11:1257-69.

25. Jarvi SI, Pitt WC, Farias ME, Shiels L, Severino MG, Howe KM, et al. Detection of Angiostrongy/us cantonensis in the blood And peripheral tissues of wild Hawaiian rats (Rattus rattus) by a quantitative PCR (qPCR) assay. PloS One. 2015;10:e0123064.

26. Qvarnstrom Y, Xayavong M, da Silva ACA, Park SY, Whelen AC, Calimlim PS, et al. Real-time polymerase chain reaction detection of Angiostrongylus cantonensis DNA in cerebrospinal fluid from 
patients with eosinophilic meningitis. Am J Trop Med Hyg. 2016;94:176-81.

27. Eamsobhana P, Wanachiwanawin D, Dechkum N, Parsartvit A, Yong HS. Molecular diagnosis of eosinophilic meningitis due to Angiostrongylus cantonensis (Nematoda: Metastrongyloidea) by polymerase chain reaction-DNA sequencing of cerebrospinal fluids of patients. Mem I Oswaldo Cruz. 2013;108:116-8.

28. Weirather JL, Jeronimo SM, Gautam S, Sundar S, Kang M, Kurtz MA, et al. Serial quantitative PCR assay for detection, species discrimination, and quantification of Leishmania spp. in human samples. J Clin Microbiol. 2011;49:3892-904.

29. Barbau-Piednoir E, Bertrand S, Mahillon J, Roosens NH, Botteldoorn N. SYBR® Green qPCR Salmonella detection system allowing discrimination at the genus, species and subspecies levels. Appl Microbiol Biotechnol. 2013;97:9811-24.

30. Lamaningao P, Kanda S, Laimanivong S, Shimono T, Darcy AW, Phyaluanglath A, et al. Development of a PCR assay for diagnosing trematode (Opisthorchis and Haplorchis) infections in human stools. Am J Trop Med Hyg. 2017;96:221-8.

31. Vitta A, Nateeworanart S, Tattiyapong M. Survey of Angiostrongylus cantonensis in rats and giant African land snails in Phitsanulok province, Thailand. Asian Pac J Trop Med. 2011;4:597-9.

32. Ash LR. Diagnostic morphology of the third-stage larvae of Angiostrongylus cantonensis, Angiostrongylus vasorum, Aelurostrongylus abstrusus, and Anafilaroides rostratus (Nematoda: Metastrongyloidea). J Parasitol. 1970:249 - 53.

33. Kibbe WA. OligoCalc: an online oligonucleotide properties calculator. Nucleic Acids Res. 2007;35(suppl_2):W43-W6.

34. Untergasser A, Nijveen H, Rao X, Bisseling T, Geurts R, Leunissen JA. Primer3Plus, an enhanced web interface to Primer3. Nucleic Acids Res. 2007;35(suppl_2):W71-W4.

35. Johnson M, Zaretskaya I, Raytselis Y, Merezhuk Y, McGinnis S, Madden TL. NCBI BLAST: a better web interface. Nucleic Acids Res. 2008;36(suppl_2):W5-9.

36. Nuñez LF, Santander-Parra SH, Chaible L, De la Torre DI, Buim MR, Murakami A, et al. Development of a Sensitive Real-Time Fast-qPCR Based on SYBR ${ }^{\circledR}$ Green for Detection and Quantification of Chicken Parvovirus (ChPV). Vet Sci. 2018;5:69.

37. Schalasta G, Arents A, Schmid M, Braun R, Enders G. Fast and type-specific analysis of herpes simplex virus types 1 and 2 by rapid PCR and fluorescence melting-curve-analysis. Infection. 2000;28:85-91.

38. Qvarnstrom Y, da Silva ACA, Teem JL, Hollingsworth R, Bishop H, Graeff-Teixeira C, et al. Improved molecular detection of Angiostrongylus cantonensis in mollusks and other environmental samples with a species-specific internal transcribed spacer 1-based TaqMan assay. Appl Environ Microbiol. 2010;76:5287-9.

39. Qvarnstrom Y, Sullivan JJ, Bishop HS, Hollingsworth R, da Silva AJ. PCR-based detection of Angiostrongylus cantonensis in tissue and mucus secretions from molluscan hosts. Appl Environ Microbiol. 2007;73:1415-9. 


\section{Figures}

(A)

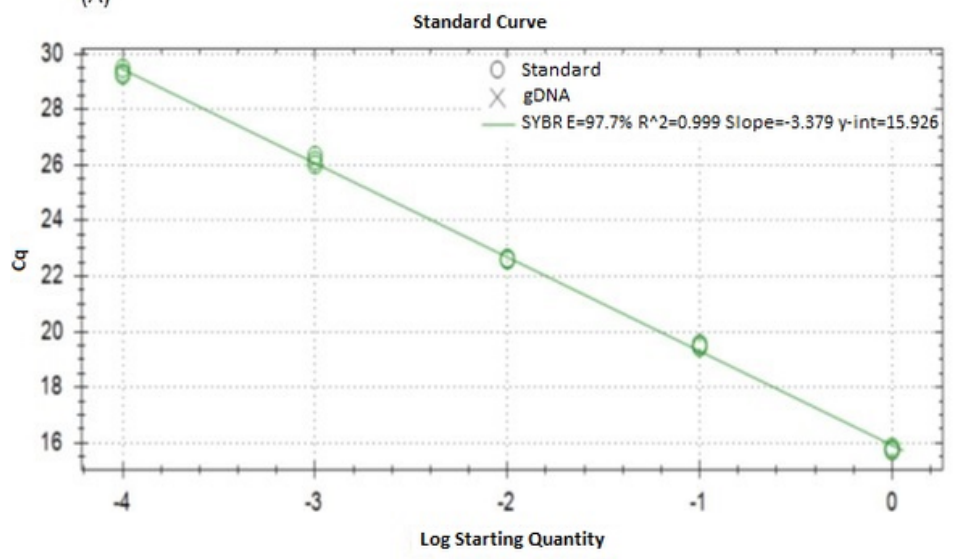

(B)

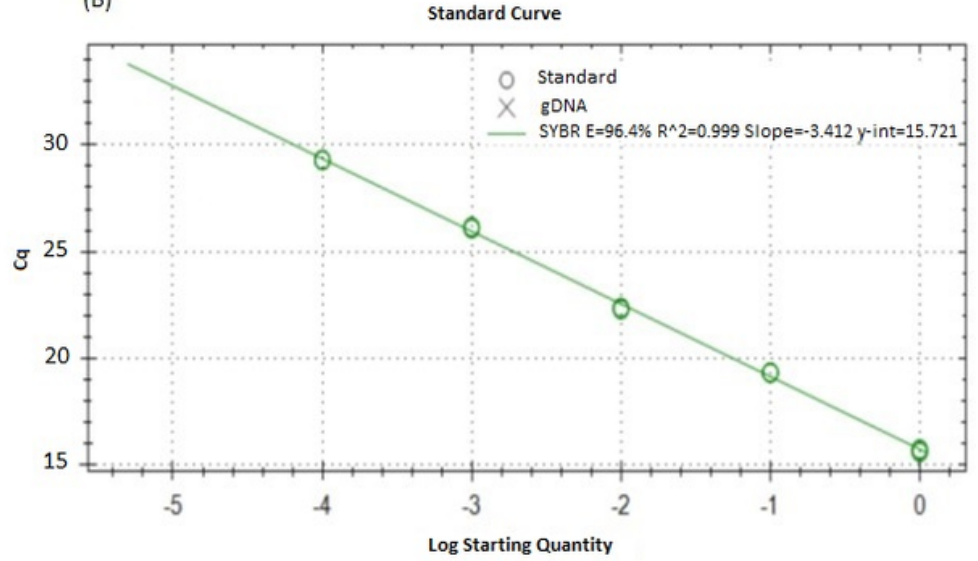

Figure 1

Amplification plots indicating the standard curve of the SYBR green qPCR with each species-specific primer for A. cantonensis (A) and A. malaysiensis (B) Cytb. The standard curves are plotted from 10-fold serial dilutions of gDNA from 10-4 to $1 \mathrm{ng} / \mu \mathrm{l}$.
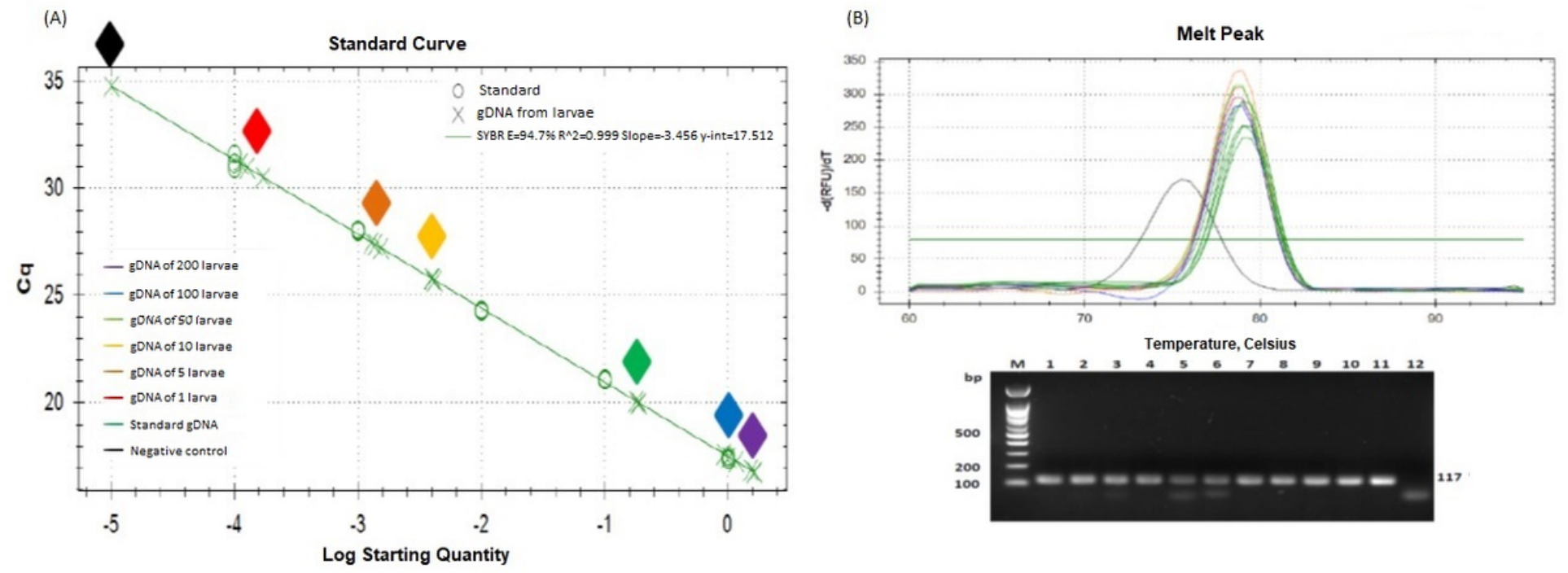

Figure 2

Sensitivity test for species-specific primers using an SYBR-green qPCR approach. (A) Quantitative estimation of the gDNA from third-stage larva of A. cantonensis. The gDNA of various larvae numbers, 1 , $5,10,50,100$, and 200, were amplified and plotted on the standard curve constructed from the 10-fold serial dilution (10-4-1 ng). (B) The melting curve of A. cantonensis was specific at 79ロC (left). Gel electrophoresis showing the results of qPCR; lanes $1-5$ are the PCR products of 10 -fold serial dilution of gDNA from 10-4 to $1 \mathrm{ng} / \mu \mathrm{l}$, respectively; lanes 6-12 are the PCR products amplified from gDNA extracted from 1, 5, 10, 50, 100, and 200 larvae and the negative control (right). 
(A)

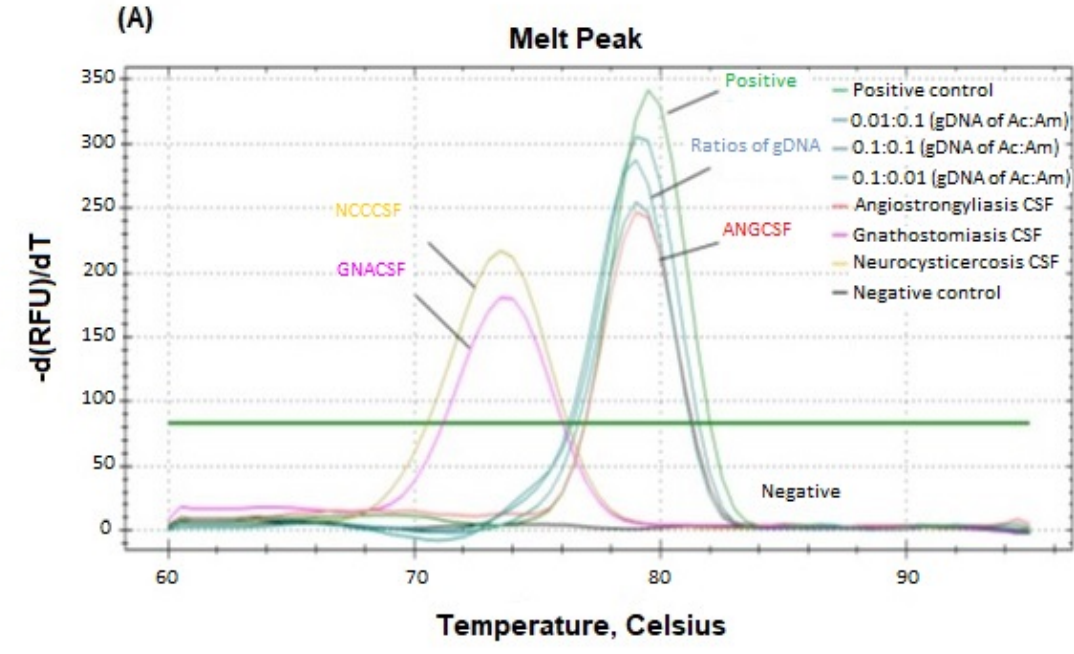

(B)

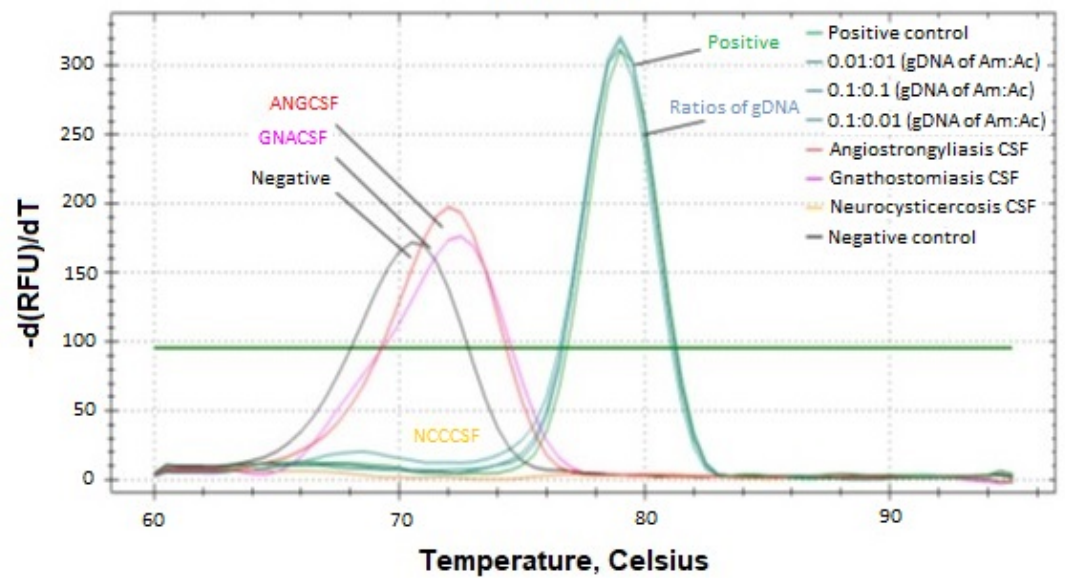

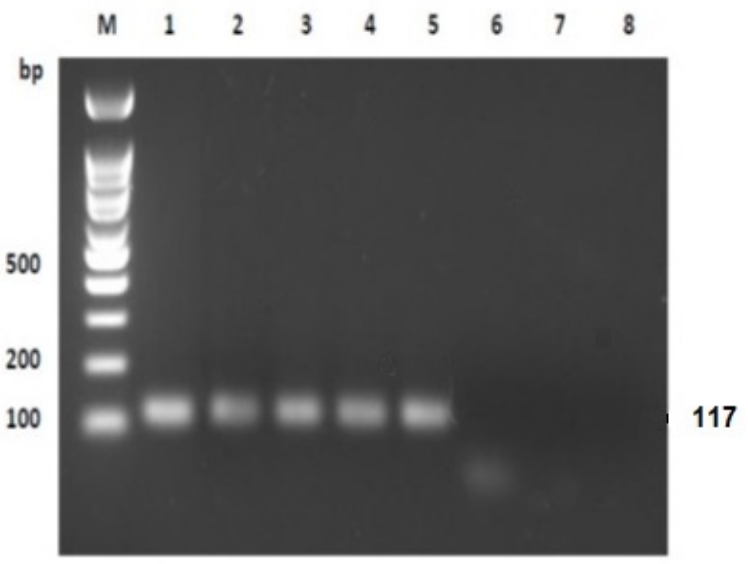

117

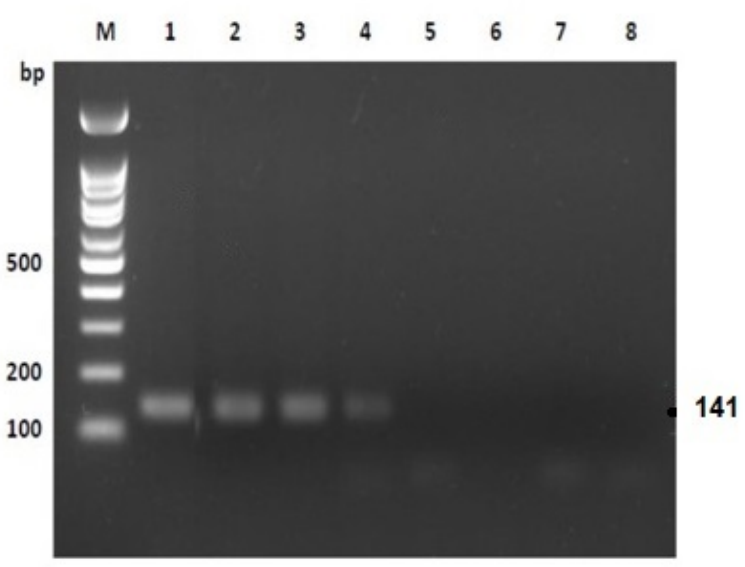

\section{Figure 3}

Specificity of species-specific primers for A. cantonensis and A. malaysiensis. (A) The specificity of AC4_cytb_F and AC5_cytb_R primers to Cytb of A. cantonensis, and the CSF from patients of gnathostomiasis (GNA), neurocysticercosis (NCC), and angiostrongyliasis (ANG) using the SYBR green qPCR method. Gel electrophoresis showing the results of SYBR-green qPCR. Lane 1 is the PCR product from $1 \mathrm{ng}$ gDNA of $A$. cantonensis. Lanes 2-4 are PCR products from the different ratios (10-2:10-1 ng, 10-1:10-1 ng, and 10-1:10-2 ng) of the gDNA of A. cantonensis and A. malaysiensis, respectively. Lane 5 is the PCR product of CSF from a patient with angiostrongyliasis. Lanes 6 and 7 show no PCR product from patients with neurocysticercosis and gnathostomiasis. Lane 8 is the negative control. (B) The specificity of AM3_cytb_F and AM4_cytb_R primers to Cytb of A. malaysiensis and the CSF from patients with gnathostomiasis (GNA), neurocysticercosis (NCC), and angiostrongyliasis (ANG) using SYBR-green qPCR. Gel electrophoresis showing the results of SYBR-green qPCR. Lane 1 is the PCR product from $1 \mathrm{ng}$ of A. malaysiensis gDNA. Lanes 2-4 are PCR products from the different ratios (10-1:10-2 ng, 10-1:10-1 ng, and 10-2:10-1 ng) of A. malaysiensis and A. cantonensis gDNA, respectively. Lanes 5-7 show no PCR product for the CSF of patients with angiostrongyliasis, neurocysticercosis, and gnathostomiasis. Lane 8 is the negative control. 


\section{Supplementary Files}

This is a list of supplementary files associated with this preprint. Click to download.

- TableS1.docx

- Tables2.docx

- FigureS1tiff300.tif

- Figures2tiff300.tif

- FigureS3tiff300.png

- CoverletterforqPCR.docx

- Graphicalabtract.jpg 